\title{
A Markov Chain on the Symmetric Group That Is Schubert Positive?
}

\author{
Thomas Lam and Lauren Williams
}

\section{CONTENTS}

1. A Markov Chain on the Symmetric Group

2. Schubert Polynomials

3. Conjectures

4. Data

Acknowledgments

References
2000 AMS Subject Classification: 05E05, 60J10

Keywords: Markov chain, Schubert polynomials, symmetric group
We study a multivariate Markov chain on the symmetric group with remarkable enumerative properties. We conjecture that the stationary distribution of this Markov chain can be expressed in terms of positive sums of Schubert polynomials. This Markov chain is a multivariate generalization of a Markov chain introduced by the first author in the study of random affine Weyl group elements.

\section{A MARKOV CHAIN ON THE SYMMETRIC GROUP}

A recent trend in algebraic combinatorics is the study of Markov chains whose stationary distributions have a combinatorial description. For example, the RazumovStroganov conjecture concerns a Markov chain on the set of link patterns on $2 n$ points around a circle; Razumov and Stroganov conjectured that each component of the stationary distribution could be described as a sum over alternating-sign matrices [Razumov and Stroganov 04]. After intensive study (see [Di Francesco et al. 06] and references therein), this conjecture was recently proved in [Cantini and Sportiello 11]. To give another example, the asymmetric exclusion process (ASEP) is a Markov chain on the $2^{n}$ words of length $n$ in two letters. Different variants of the ASEP were studied in [Duchi and Schaeffer 05] and [Corteel and Williams 07, Corteel and Williams 12], in which the stationary distributions were described in terms of Dyck paths, permutations, and staircase tableaux.

In this paper, we study a Markov chain on the symmetric group whose stationary distribution appears to have remarkable combinatorial properties. Let $S_{n}, n \geq 3$, denote the symmetric group on $n$ letters and let $(i, j)$ denote the transposition that swaps $i$ and $j$. We use conventions so that left multiplication acts on values and right multiplication acts on positions. 
Define a matrix $P=\left(p_{w, v}\right)_{w, v \in S_{n}}$ by

$$
p_{w, v}= \begin{cases}x_{w^{-1}(i+1)} & \text { if } v=(i, i+1) w<w, \\ x_{w^{-1}(1)} & \text { if } v=(1, n) w>w, \\ * & \text { if } w=v, \\ 0 & \text { otherwise, }\end{cases}
$$

where $*$ is chosen so that $\sum_{v \in S_{n}} p_{w, v}=1$ for each $w \in$ $S_{n}$. If the $x_{i}$ are nonnegative real numbers summing to at most 1 , then we can think of $P$ as defining a Markov chain on $S_{n}$.

When we set $x_{i}=1 / n$, we obtain the Markov chain defined in [Lam 11, Section 3]. This specialized Markov chain $\left.P\right|_{1 / n}$ was introduced to study the asymptotic behavior of random elements in the affine symmetric group, or equivalently, random walks in the affine braid arrangement. The stationary distribution of $\left.P\right|_{1 / n}$ was shown to control the asymptotic "shapes" of random affine symmetric group elements. In particular, a detailed understanding of the Markov chain $P$ would have applications to the problem studied in [Lam 11] - our conjectures here imply [Lam 11, Conjecture 1].

Proposition 1.1. The matrix $P^{T}-I$ has a onedimensional nullspace for generic values of $x$. In particular, when the $x_{i}$ are nonnegative real numbers summing to at most 1 , the Markov chain defined by $P$ has a unique stationary distribution.

Proof. When all $x_{i}$ are positive and sum to at most 1, then it follows from [Lam 11, Proposition 1] that we have an irreducible and aperiodic Markov chain on $S_{n}$, and thus we have a unique invariant distribution. If we treat $x_{1}, \ldots, x_{n-2}, x_{n-1}$ as variables, then a basis of the nullspace of $P^{T}-I$ can be written as a rational function in the $x_{i}$. This nullspace must be one-dimensional.

Let $\left\{\zeta(w) \in \mathbb{Q}\left(x_{1}, x_{2}, \ldots, x_{n-1}\right) \mid w \in W\right\}$ denote a vector spanning the nullspace of Proposition 1.1, which we normalize by setting

$$
\zeta\left(w_{0}\right)=x_{1}^{1+2+\cdots+n-2} x_{2}^{1+2+\cdots+n-3} \cdots x_{n-2} .
$$

Suppose $w=w_{1} w_{2} \cdots w_{n} \in S_{n}$. Let

$$
\chi(w)=\left(w_{1}+1\right)\left(w_{2}+1\right) \cdots\left(w_{n}+1\right) \in S_{n}
$$

be the cyclic shift of $w$, where the letters of $\chi(w)$ are interpreted modulo $n$. The following is derived immediately from the definitions.

Proposition 1.2. For each $w \in W$, we have $\zeta(\chi(w))=$ $\zeta(w)$.

\section{SCHUBERT POLYNOMIALS}

We fix notation concerning Schubert polynomials. Let $\partial_{i}$ denote the divided difference operator on polynomials in $x_{1}, x_{2}, \ldots$, defined by

$$
\begin{aligned}
\partial_{i} f & \left(x_{1}, x_{2}, \ldots\right) \\
& =\frac{f\left(x_{1}, \ldots, x_{i}, x_{i+1}, \ldots\right)-f\left(x_{1}, \ldots, x_{i+1}, x_{i}, \ldots\right)}{x_{i}-x_{i+1}} .
\end{aligned}
$$

For the longest permutation $w_{0} \in S_{n}$, we first define

$$
\mathfrak{S}_{w_{0}}\left(x_{1}, x_{2}, \ldots\right):=x_{1}^{n-1} x_{2}^{n-2} \cdots x_{n-1} .
$$

Next for $w \in S_{n}$, we let $w^{-1} w_{0}=s_{i_{1}} s_{i_{2}} \cdots s_{i_{\ell}}$ be a reduced expression. Then

$$
\mathfrak{S}_{w}:=\partial_{i_{1}} \partial_{i_{2}} \cdots \partial_{i_{\ell}} \mathfrak{S}_{w_{0}}
$$

The polynomial $\mathfrak{S}_{w}$ does not depend on the choice of the reduced expression. Furthermore, $\mathfrak{S}_{w}$ does not depend on the symmetric group to which $w$ is considered to belong.

\section{CONJECTURES}

Our main conjecture is the following.

Conjecture 3.1. In increasing strength:

1. Each $\zeta(w)$ is a polynomial.

2. Each $\zeta(w)$ is a polynomial with nonnegative integer coefficients.

3. Each $\zeta(w)$ is a nonnegative integral sum of Schubert polynomials $\mathfrak{S}_{u}\left(x_{1}, x_{2}, \ldots\right)$.

Let $\eta(w)$ denote the largest monomial that can be factored out of $\zeta(w)$. By Proposition 1.2, $\eta(w)=\eta(\chi(w))$. Write $[m]$ to denote $\{0,1,2, \ldots, m\}$.

Conjecture 3.2. (Monomial factor.) Assume Conjecture 3.1(1). The map $w \mapsto \eta(w)$ is an $n$-to-1 map from $S_{n}$ to

$$
\begin{aligned}
& \left\{x_{1}^{a_{1}+a_{2}+\cdots+a_{n-2}} x_{2}^{a_{2}+\cdots+a_{n-2}} \cdots x_{n-2}^{a_{n-2}} \mid\left(a_{1}, a_{2}, \ldots, a_{n-2}\right)\right. \\
& \in[n-2] \times[n-3] \times \cdots \times[1]\} .
\end{aligned}
$$

Moreover, $\eta(w)=x_{1}^{a_{1}+a_{2}+\cdots+a_{n-2}} x_{2}^{a_{2}+\cdots+a_{n-2}} \cdots x_{n-2}^{a_{n-2}}$ is given by

$$
a_{i}=\#\left\{k \in[i+2, n] \mid w_{k} \in\left[w_{i}, w_{i+1}\right]\right\},
$$

where $\left[w_{i}, w_{i+1}\right]$ denotes a cyclic subinterval of $[n]$. 


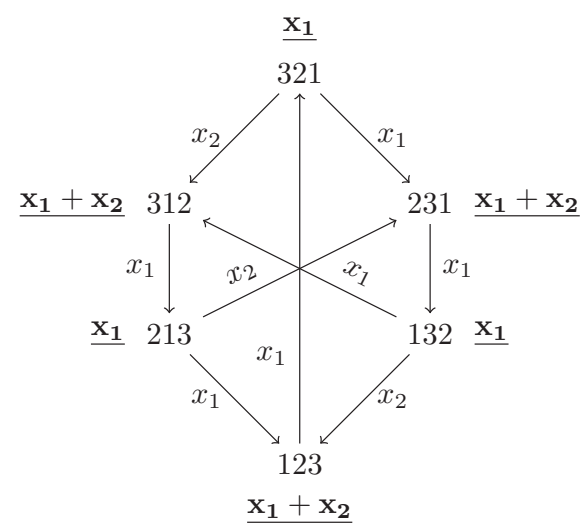

FIGURE 1. The transition matrix on $S_{3}$ (in normal font) with the transitions from a vertex to itself removed and the normalized stationary distribution $\zeta$ (in bold and underlined).

Conjecture 3.3. (Special value.) We have the following special value:

$$
\begin{aligned}
& \zeta(\text { id }) \\
& \quad=\mathfrak{S}_{123 \cdots n} \mathfrak{S}_{1 n 23 \cdots(n-1)} \mathfrak{S}_{1(n-1) n 23 \cdots(n-2)} \cdots \mathfrak{S}_{134 \cdots n 2} .
\end{aligned}
$$

Conjecture 3.4. (Special Schubert factors.) Consider the letters of $w \in S_{n}$ in (cyclic) order. If there is an adjacent string of letters 1,2 , then $\zeta(w)$ is a multiple of the Schubert polynomial $\mathfrak{S}_{1345 \ldots n 2}$. More generally, if there is an adjacent string of letters $1,2,3, \ldots, k$, then $\zeta(w)$ is a multiple of the Schubert polynomial $\mathfrak{S}_{1(k+1)(k+2) \ldots n 23 \ldots k}$.

\section{DATA}

We provide experimental data supporting these conjectures.

\subsection{The Case $n=3$}

See Figure 1.

\begin{tabular}{|c|c|c|}
\hline$w$ & $\zeta(w)$ & \\
\hline 4123 & $\left(a^{2}+a b+b^{2}\right)(a b+a c+b c)$ & $\mathfrak{S}_{1423} \mathfrak{S}_{1342}$ \\
4132 & $\left(a^{2}+a b+b^{2}\right) a b$ & $\mathfrak{S}_{1423} \mathfrak{S}_{231}$ \\
4213 & $(a+b+c) a^{2} b$ & $\mathfrak{S}_{1243} \mathfrak{S}_{321}$ \\
4231 & $\left(a^{2} b+a^{2} c+a b^{2}+a b c+b^{2} c\right) a$ & $\mathfrak{S}_{1432} \mathfrak{S}_{21}$ \\
4312 & $(a b+a c+b c) a^{2}$ & $\mathfrak{S}_{1342} \mathfrak{S}_{312}$ \\
4321 & $a^{3} b$ & $\mathfrak{S}_{4213}$ \\
\hline
\end{tabular}

TABLE 1. The case $\mathrm{n}=4$.

\begin{tabular}{|c|c|}
\hline$w$ & $\zeta(w)$ \\
\hline 51234 & $\mathfrak{S}_{15234} \mathfrak{S}_{14523} \mathfrak{S}_{13452}$ \\
51243 & $\mathfrak{S}_{15234} \mathfrak{S}_{14523} a b c$ \\
51324 & $\mathfrak{S}_{15234} \mathfrak{S}_{12453} a^{2} b^{2} c$ \\
51342 & $\mathfrak{S}_{15234} \mathfrak{S}_{14532} a b$ \\
51423 & $\mathfrak{S}_{15234} \mathfrak{S}_{13452} a^{2} b^{2}$ \\
51432 & $\mathfrak{S}_{15234} a^{3} b^{3} c$ \\
52134 & $\mathfrak{S}_{12534} \mathfrak{S}_{13452} a^{3} b^{2}$ \\
52143 & $\mathfrak{S}_{12534} a^{4} b^{3} c$ \\
52314 & $\left(\mathfrak{S}_{15432}+\mathfrak{S}_{164235}\right) a^{2} b c$ \\
52341 & $\left(\mathfrak{S}_{1753246}+\mathfrak{S}_{265314}+\mathfrak{S}_{2743156}\right.$ \\
& $\left.+\mathfrak{S}_{356214}+\mathfrak{S}_{364215}+\mathfrak{S}_{365124}\right) a$ \\
52413 & $\left(\mathfrak{S}_{164325}+\mathfrak{S}_{25431}\right) a^{2} b$ \\
52431 & $\mathfrak{S}_{15243} a^{3} b^{2} c$ \\
53124 & $\left(\mathfrak{S}_{146325}+\mathfrak{S}_{24531}\right) a^{3} b$ \\
53142 & $\mathfrak{S}_{12543} a^{4} b^{2} c$ \\
53214 & $\mathfrak{S}_{12354} a^{5} b^{3} c$ \\
53241 & $\mathfrak{S}_{13542} a^{4} b^{2}$ \\
53412 & $\mathfrak{S}_{15423} \mathfrak{S}_{13452} a^{2}$ \\
53421 & $\mathfrak{S}_{15423} a^{3} b c$ \\
54123 & $\mathfrak{S}_{14523} \mathfrak{S}_{13452} a^{3}$ \\
54132 & $\mathfrak{S}_{14523} a^{4} b c$ \\
54213 & $\mathfrak{S}_{12453} a^{5} b^{2} c$ \\
54231 & $\mathfrak{S}_{14532} a^{4} b$ \\
54312 & $\mathfrak{S}_{13452} a^{5} b^{2}$ \\
54321 & $a^{6} b^{3} c$ \\
\hline & \\
\hline
\end{tabular}

TABLE 2. The case $\mathrm{n}=5$.

\subsection{The Case $n=4$}

Using Proposition 1.2, we see that we need to provide data only for permutations $w$ for which $w_{1}=n$. In Table 1 , we use $a=x_{1}, b=x_{2}$, and $c=x_{3}$. We also write the answers as products of Schubert polynomials. Since a product of Schubert polynomials is also a nonnegative linear combination of Schubert polynomials, this supports Conjecture 3.1(3).

Note that $a^{2} b+a^{2} c+a b^{2}+a b c+b^{2} c$ is the only nontrivial factor that is not a symmetric polynomial.

\subsection{The Case $n=5$}

For $n=5$, we write our answers as products and sums of Schubert polynomials, multiplied by the monomial factor $\eta(w)$. See Table 2 .

\section{ACKNOWLEDGMENTS}

Thomas Lam was supported by NSF grant DMS-0901111 and by a Sloan Fellowship. Lauren Williams was supported by NSF grant DMS-0854432 and by a Sloan Fellowship. 


\section{REFERENCES}

[Cantini and Sportiello 11] L. Cantini and A. Sportiello. "Proof of the Razumov-Stroganov Conjecture." J. Comb. Theory A 118 (2011), 1549-1574.

[Corteel and Williams 07] S. Corteel, L. Williams. "A Markov Chain on Permutations Which Projects to the PASEP (Partially Asymmetric Exclusion Process)." Int. Math. Res. Not. 2007, article ID mm055.

[Corteel and Williams 12] S. Corteel and L. Williams. "Tableaux Combinatorics for the Asymmetric Exclusion Process and Askey-Wilson Polynomials." To appear in Duke Math. J. 159 (2011), 385-415.

[Di Francesco et al. 06] P. Di Francesco, P. Zinn-Justin, and J. B. Zuber. "Sum Rules for the Ground States of the
$O(1)$ Loop Model on a Cylinder and the XXZ Spin Chain." J. Stat. Mech. (2006), P08011.

[Duchi and Schaeffer 05] E. Duchij and G. Schaeffer. "A Combinatorial Approach to Jumping Particles." J. Combin. Theory Ser. A 110 (2005), 1-29.

[Lam 11] T. Lam. "The Shape of a Random Affine Weyl Group Element and Random Core Partitions." Preprint, 2011.

[Razumov and Stroganov 04] A. Razumov and Y. Stroganov. "Combinatorial Nature of Ground State Vector of $O(1)$ Loop Model." Theor. Math. Phys. 138 (2004), 333-337, 395-400.

Thomas Lam, Department of Mathematics, University of Michigan, Ann Arbor, MI 48109, USA (tfylam@umich.edu)

Lauren Williams, Department of Mathematics, University of California, Berkeley, CA 94705, USA

(williams@math.berkeley.edu)

Received March 1, 2011; accepted March 29, 2011 